\title{
Utility of Magnetic Resonance Imaging-Based Finite Element Analysis for the Biomechanical Stress Analysis of Hemorrhagic and Non-Hemorrhagic Carotid Plaques
}

\author{
Umar Sadat, MD; Zhongzhao Teng, PhD; Victoria E. Young, MD; \\ Zhi Yong Li, PhD; Jonathan H. Gillard, MD
}

\begin{abstract}
Background: Biomechanical stress analysis has been used for plaque vulnerability assessment. The presence of plaque hemorrhage $(\mathrm{PH})$ is a feature of plaque vulnerability and is associated with thromboembolic ischemic events. The purpose of the present study was to use finite element analysis (FEA) to compare the stress profiles of hemorrhagic and non-hemorrhagic profiles.
\end{abstract}

\begin{abstract}
Methods and Results: Forty-five consecutive patients who had suffered a cerebrovascular ischemic event with an underlying carotid artery disease underwent high-resolution magnetic resonance imaging (MRI) of their symptomatic carotid artery in a 1.5-T MRI system. Axial images were manually segmented for various plaque components and used for FEA. Maximum critical stress (M-CstresssL) for each slice was determined. Within a plaque, the maximum M-CstresssL for each slice of a plaque was selected to represent the maximum critical stress of that plaque (M-CstresSPL) and used to compare hemorrhagic and non-hemorrhagic plaques. A total of $62 \%$ of plaques had hemorrhage. It was observed that plaques with hemorrhage had significantly higher stress (M-CstresSPL) than plaques without $\mathrm{PH}$ (median [interquartile range]: $315 \mathrm{kPa}$ [247-434] vs. $200 \mathrm{kPa}$ [171-282], P=0.003).
\end{abstract}

Conclusions: Hemorrhagic plaques have higher biomechanical stresses than non-hemorrhagic plaques. MRIbased FEA seems to have the potential to assess plaque vulnerability. (Circ J 2011; 75: 884-889)

Key Words: Atherosclerosis; Biomechanical stresses; Finite element analysis; Magnetic resonance imaging; Stroke

$\mathbf{P}$ laque hemorrhage $(\mathrm{PH})$ is a common feature of complex atheromatous plaques. It is associated with thromboembolic (ischemic) events in coronary ${ }^{1}$ and carotid arteries. $^{2-4}$ The importance of $\mathrm{PH}$ was suggested by Imparato et al, who reported, in 1979, that PH was responsible for $73 \%$ of focal neurological symptoms in patients with symptomatic carotid artery disease and that it played a significant role in plaque progression. ${ }^{5}$ Similar results have been reported by Takaya et al in a longitudinal magnetic resonance imaging (MRI) study. ${ }^{6}$ A recent systematic review (of 31 observational studies) also suggested a plausible contribution of $\mathrm{PH}$ to focal neurological symptom production. ${ }^{7}$ Because the location of carotid plaque is in a dynamic environment at the carotid bifurcation, the relationship between plaque morphology and biomechanical stresses cannot be overlooked. It was Lusby et al in the 1980s who suggested that mechanical stresses played a role in carotid plaque progression and in the development of symptoms of cerebral ischemia. ${ }^{8,9}$ The stress profiles of hemorrhagic and non-hemorrhagic plaques, however, remain unexplored.

\section{Editorial p 783}

Finite element analysis (FEA) is used in the biomechanical stress analysis of plaques and has been used to show differences between stress profiles of symptomatic and asymptomatic plaques, ${ }^{10}$ and also in subgroups of symptomatic plaques. ${ }^{11}$ Recently we demonstrated that high biomechanical structural stresses are associated with subsequent cerebrovascular events in patients with mild-moderate carotid stenosis. ${ }^{12} \mathrm{We}$ have also recently reported the effectiveness of biomechanical stress analysis for plaque burden-based stress analysis of acutely symptomatic and asymptomatic patients. ${ }^{13}$ Because biomechanical stress analysis of plaques incorporates information about plaque morphology, material properties of various plaque components and local hemodynamic factors, it has the potential to provide a comprehensive assessment of the vulnerability status of plaques for the identification of high-

Received July 22, 2010; revised manuscript received November 7, 2010; accepted November 26, 2010; released online February 2, 2011 Time for primary review: 48 days

University Department of Radiology, University of Cambridge, Cambridge (U.S., Z.T., V.E.Y., Z.Y.L., J.H.G.); Cambridge Vascular Unit, Cambridge University Hospitals NHS Foundation Trust, Cambridge (U.S.), UK

Mailing address: Umar Sadat, MD, Box 218, Level 5, University Department of Radiology, Addenbrooke's Hospital, Hills Road, Cambridge CB2 0QQ, UK. E-mail: us229@cam.ac.uk

ISSN-1346-9843 doi:10.1253/circj.CJ-10-0719

All rights are reserved to the Japanese Circulation Society. For permissions, please e-mail: cj@j-circ.or.jp 

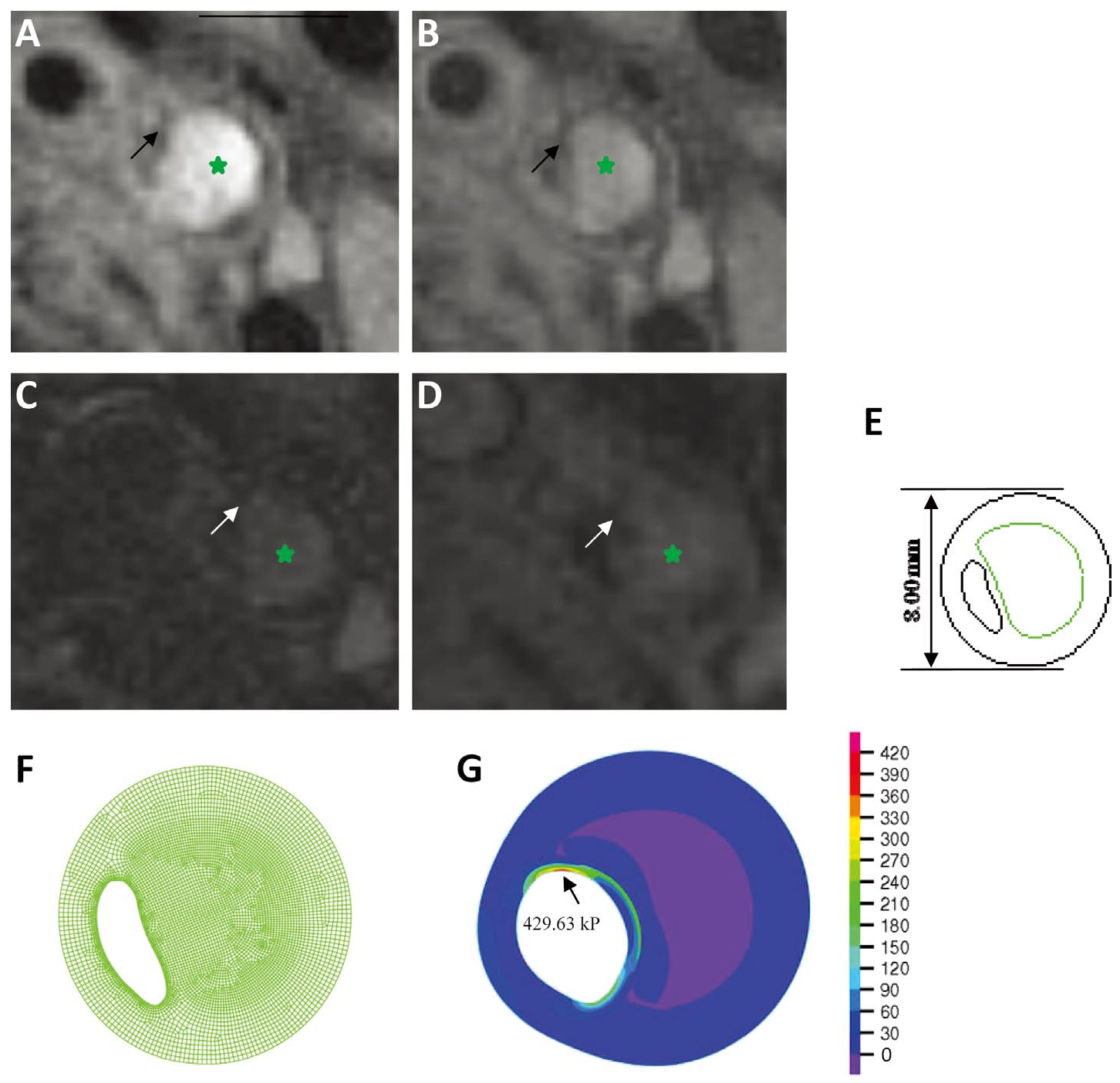

Figure 1. (A) $\mathrm{T}_{1-}$, (B) proton density-, (C) $\mathrm{T}_{2}$ - and (D) short $\tau$ inversion recovery (STIR)-weighted magnetic resonance imaging showing fresh plaque hemorrhage (green star), which is hyperintense on T1-weighted imaging and isointense on T2- and STIRweighted imaging (white arrow, rupture). (E) Contours as exported into the simulation software with green contour indicating the plaque hemorrhage. (F) Fine mesh generated by ADINA 8.6 used for simulation (measurement of element edge was at least $<0.1 \mathrm{~mm}$ for the plaque components and $<0.05 \mathrm{~mm}$ at the lumen boundary to avoid computational artifact). (G) Band plot showing critical stress concentration at the vulnerable site (plaque shoulder in this case).

risk patients. In the present study we performed biomechanical stress profiling of hemorrhagic and non-hemorrhagic plaques.

\section{Methods}

Forty-five consecutive patients who had suffered a cerebrovascular ischemic event underwent high-resolution MRI of their symptomatic carotid arteries within $72 \mathrm{~h}$ of the acute event. A retinal transient ischemic attack (TIA) was defined as a partial or complete visual field loss of ischemic origin in 1 eye lasting $<24 \mathrm{~h}$, and a hemispheric TIA was defined as a focal cerebral dysfunction of ischemic origin lasting $<24 \mathrm{~h} .{ }^{14}$ A minor stroke was defined as a neurological dysfunction of ischemic origin with deficits persisting $>24 \mathrm{~h}$ but with a Rankin score $\leq 3$. The neurological assessment was done by an experienced stroke physician. The study protocol was reviewed and approved by the regional research ethics committee and all patients gave written informed consent.

The criteria for inclusion in the study were (1) internal carotid artery stenosis of $\geq 30-99 \%$ on duplex imaging during screening assessment; (2) sufficient MRI image quality to identify the lumen wall and outer boundary of the arterial wall (the image quality [IQ] was rated before review using a previously published 5-point scale, ${ }^{15,16}$ and images with IQ $>3$ were included for morphological analysis); and (3) normal heart rhythm, confirmed on 24-h Holter monitoring and normal transthoracic echocardiography in patients in whom a cause of stroke other than carotid artery disease was suspected.

Exclusion criteria included (1) previous carotid endarterectomy of the symptomatic carotid artery; (2) cardiac arrhythmias; (3) known coagulation/clotting disorder responsible 

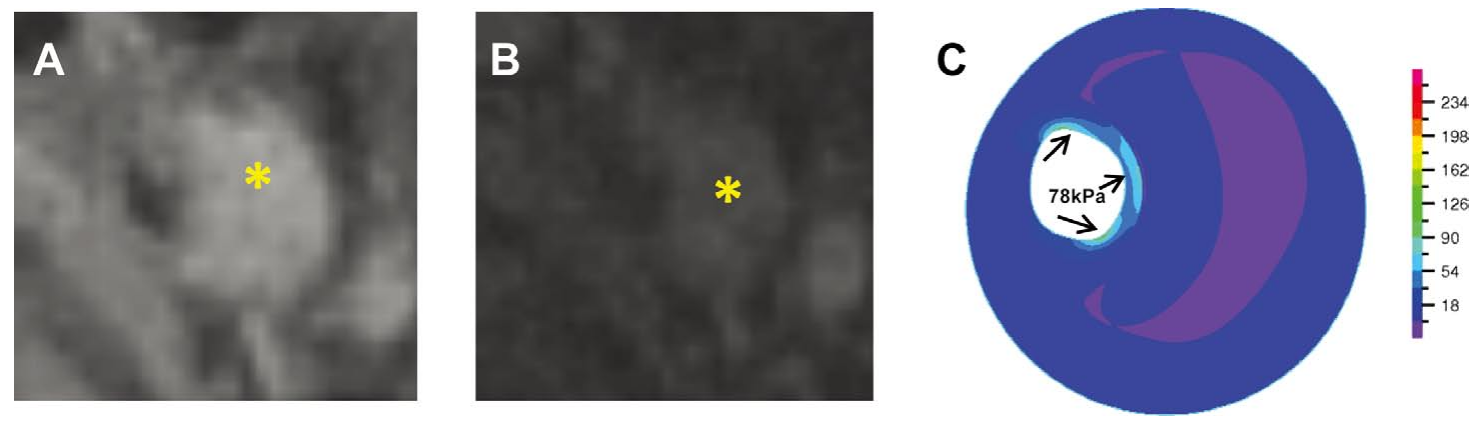

Figure 2. Lipid core (yellow star), which is hyperintense on (A) T1-weighted imaging and hypointense on (B) T2-weighted imaging. (C) Band plot showing critical stress concentration.

for the symptoms; (4) thrombolysis following the acute cerebrovascular event; and (5) clinical contraindications to MRI, for example inner ear implants, pacemaker, and so on.

\section{High-Resolution Multi-Contrast MRI}

High-resolution carotid MRI of the research subjects was performed using a 1.5-T MRI system (Signa HDx GE Healthcare, Waukesha, WI, USA) with a 4-channel phased-array neck coil (PACC, Machnet BV, Elde, The Netherlands). Movement artifact was minimized using a dedicated vacuum-based head restraint system (VAC-LOK Cushion, Oncology Systems Limited, UK) to fix the head and neck in a comfortable position and allow close apposition of the surface coils. After an initial coronal localizer sequence, axial 2-D time-of-flight (TOF) MR angiography was performed to identify the location of the carotid bifurcation and the region of maximum stenosis on each side. Three-mm-thick axial images were acquired from the carotid bifurcation for $12 \mathrm{~mm}$ below the carotid bifurcation to a point $12 \mathrm{~mm}$ distal to it to ensure that the entire plaque was imaged. ${ }^{17}$

The following electrocardiograph-gated fast spin echo pulse sequences were used to delineate various plaque components such as fibrous cap (FC), lipid-rich necrotic core (LRNC) and $\mathrm{PH}$ (Figures 1,2): T1-weighted (repetition time/echo time: $1 \times \mathrm{RR} / 7.8 \mathrm{~ms}$ ) with fat saturation; proton density (PD)weighted (repetition time/echo time: $2 \times \mathrm{RR} / 7.8 \mathrm{~ms}$ ) with fat saturation; $\mathrm{T}_{2}$-weighted (repetition time/echo time: $2 \times \mathrm{RR} /$ $85 \mathrm{~ms}$ ) with fat saturation; and short $\tau$ inversion recovery (STIR; repetition time/echo time/inversion time: $2 \times \mathrm{RR} / 42 \mathrm{~ms} /$ $150 \mathrm{~ms}$ ). The number of acquired slices for an individual plaque depended on the plaque length.

\section{MRI Analysis}

Plaque components (ie, LRNC, fibrous tissue and PH) were manually delineated by an experienced MRI reader (U.S.) using CMR Tools (London, UK) and confirmed by a consultant neuroradiologist (J.H.G.) using previously published criteria. ${ }^{18,19}$ Briefly, LRNC appears to be hyperintense on $\mathrm{T}_{1-}$ and PD-weighted images, and hypointense on STIR and $\mathrm{T}_{2}$-weighted images. Fibrous tissue appears isointense-hypointense on $\mathrm{T}_{1}$ - and PD-weighted images, and hyperintense on STIR and $\mathrm{T}_{2}$-weighted images. ${ }^{20}$ Calcium appears hypointense on all MR weightings. PH (when fresh as was the case in the present patients, being imaged within $72 \mathrm{~h}$ of acute event) appears hyperintense on $\mathrm{T}_{1}$ - and PD-weighted images, and isointense-hypointense on STIR and T2-weighted images. Following the plaque segmentation, the segmented contours were used for mesh generation.

\section{Biomechanical Stress Modeling}

The investigators responsible for the entire computational analysis were not involved in the acquisition of MRI data or image segmentation.

\section{Pre-Shrink Process for In Vivo Data}

In vivo MRI was done in diastole using cardiac gating. Therefore, a pre-shrink process was necessary to obtain the zeropressure geometry, which was used as the numerical starting geometry, and to recover the in vivo diastolic plaque geometry when pressure was imposed in the lumen. ${ }^{21}$ The average lumen circumference shrinkage was $9.75 \pm 1.86 \%$ for the 45 patients.

\section{Mesh Generation, Computational Models and Solution Methods}

The computational mesh was made and the model was solved using ADINA8.6 (ADINA, MA, USA; Figures 1F,2). All plaque components including fibrous tissue, hemorrhage, lipid core, calcification and healthy arterial wall were assumed to be non-linear, isotropic, incompressible and hyper-elastic. ${ }^{22}$ The modified Mooney-Rivlin strain energy density function was used. A pulsating pressure was imposed on the lumen, using the systolic/diastolic arm pressure data for each patient at the time of the MRI, to perform patient-specific simulations. ${ }^{23}$ Material parameters were used from available data. ${ }^{24}$ Pressure at the out-boundary of each vessel slice was set to zero. The reproducibility of the biomechanical stress simulations has been determined previously by our group. ${ }^{25}$

\section{Definition and Calculation of Critical Stress}

In continuum mechanics, stress is a measure of the average force per unit area of a surface within a deformable body on which internal forces act. The maximum critical stress was defined as the maximum principal stress at vulnerable plaque sites, that is, plaque shoulder and minimum FC thickness. ${ }^{26}$ The "maximum critical stress" of a slice is indicated as MCStresssL throughout the text. To obtain the maximum critical stress of the entire plaque (M-CStresspL), the maximum M-CStresssz for each slice of a plaque was selected.

\section{Statistical Analysis}

The normality of the data was assessed on Shapiro-Wilk nor- 


\begin{tabular}{|c|c|c|c|}
\hline & $\begin{array}{l}\text { Non-hemorrhagic patients } \\
\qquad(n=17)\end{array}$ & $\begin{array}{l}\text { Hemorrhagic patients } \\
\qquad(n=28)\end{array}$ & $P$ value \\
\hline Age (years) (SD) & $75(9)$ & $73(13)$ & 0.98 \\
\hline Hypertension & $6(35)$ & $17(60)$ & 0.12 \\
\hline Diabetes & $2(12)$ & $3(11)$ & 1.00 \\
\hline Peripheral vascular disease & $1(6)$ & $5(18)$ & 0.38 \\
\hline Ischemic heart disease & $7(41)$ & $11(39)$ & 1.00 \\
\hline Coronary artery bypass graft & $1(6)$ & $4(14)$ & 0.63 \\
\hline Aspirin & $10(59)$ & $14(50)$ & 0.75 \\
\hline Statins & $12(70)$ & $20(71)$ & 1.00 \\
\hline Clopidogrel & $0(0)$ & $2(7)$ & 0.51 \\
\hline ACEI & $5(29)$ & $6(21)$ & 0.72 \\
\hline ECST \% carotid stenosis (SD) & $49(14)$ & $55(14)$ & 0.18 \\
\hline Systolic blood pressure $(\mathrm{mmHg})$ & $144 \pm 26$ & $140 \pm 20$ & 0.44 \\
\hline Diastolic blood pressure $(\mathrm{mmHg})$ & $84 \pm 10$ & $80 \pm 14$ & 0.30 \\
\hline FC ruptures & $10(22)$ & $23(51)$ & 0.10 \\
\hline Large lipid core $n(\%)$ & $4(9)$ & $10(22)$ & 0.51 \\
\hline
\end{tabular}

Data given as mean \pm SD or $n(\%)$

ACEl, angiotensin-converting enzyme inhibitors; FC, fibrous cap.

mality test. Categorical variables were assessed on Fisher's exact test while continuous variables were assessed on MannWhitney test for non-parametric data and unpaired Student's t-test for parametric data. The level of statistical significance was set at $<0.05$. All statistical analyses were performed in GraphPad Instat (Version: 3.06).

\section{Results}

The patient demographics and comorbidities are summarized in Table. There was no statistically significant difference between hemorrhagic and non-hemorrhagic plaques regarding prevalence of FC rupture and the presence of large lipid cores (lipid core occupying $>25 \%$ of plaque volume ${ }^{27}$ ). In total, 173 MRI slices were analyzed for 45 carotid plaques. All slices used for analysis had image quality $>3$. Sixty-two percent of plaques $(n=28)$ had MRI evidence of $\mathrm{PH}$ compared to $38 \%(n=17)$ without hemorrhage. The hemorrhage within all plaques was identifiable as fresh PH on MRI (Figure 1). It was found that the M-CStresspl of hemorrhagic plaques was significantly higher than that of non-hemorrhagic plaques (Figure 2; median [interquartile range]: $315 \mathrm{kPa}$ [247-434] vs. $200 \mathrm{kPa}$ [171-282], $\mathrm{P}=0.003)$.

\section{Discussion}

To our knowledge this is the first study to provide a snapshot of the biomechanical stresses within vulnerable atheromatous plaques in the presence of $\mathrm{PH}$ compared to those without $\mathrm{PH}$. We found that hemorrhagic plaques had significantly higher biomechanical stresses than non-hemorrhagic plaques. Although it remains unknown whether PH leads to high stresses or vice versa, in biomechanical terms, high intrinsic mechanical stresses would result because of the soft nature of $\mathrm{PH}$ and its ability to undergo massive deformation when loading force is applied to it. In the dynamic environment of the carotid artery bifurcation this leads to extensive plaque morphology disruption and reconfiguration, as suggested by Lusby et al.,3 This accounts for enhanced plaque vulnerability and predisposes to recurrent focal neurological symptoms in patients with mild, moderate and severe carotid stenosis. From the pathological viewpoint, the disruption of neovessels within advanced atheromatous plaques resulting from underlying high inflammatory activity, leads to the oozing of blood from the leaky vessels resulting in hemorrhagic transformation of the plaque. The present observation that both groups had comparable prevalence of FC rupture despite the predominant presence of $\mathrm{PH}$ in 1 group supports the fact that $\mathrm{PH}$ results from the oozy neovessels within the plaque rather than from FC rupture. The transition from an asymptomatic state to a symptomatic state is rapid and often unpredictable. ${ }^{28}$ The sudden occurrence of hemorrhage within plaques most likely results in sudden changes in the biomechanical properties of the plaque, making it rupture prone. Hemoglobin released from the red blood cells at the site of the hemorrhage also plays a large part in destabilizing the plaque because of its potent pro-inflammatory nature, thereby promoting the formation of reactive oxygen species. ${ }^{29}$ All these factors, in addition to turbulent flow, changes in vessel wall stiffness due to atheroma, segmental variations in vessel wall distensibility and wall compliance, ${ }^{30}$ and alterations in segmental tangential tension due to the asymmetric deposition of plaque, ${ }^{9}$ generate a high-risk environment within hemorrhagic plaques, making them extremely dangerous.

Strong evidence of the association of $\mathrm{PH}$ with plaque instability comes from the Oxford Plaque Study, a recent largescale study of 526 symptomatic carotid plaques. It was found that in $65 \%$ of patients whose carotid plaques were removed sooner than 60-90 days after the most recent ischemic event or symptom onset, histological features of $\mathrm{PH}$ were present. ${ }^{31}$ The prevalence of $\mathrm{PH}$ in the present patients was $62 \%$, which is in accordance with the aforementioned study and with our previous report. ${ }^{19}$

In the present study FEA was used for the biomechanical stress simulations. We have used this method extensively in the past to assess features of plaque vulnerability and compare different patient groups. ${ }^{10,11,32-38}$ The principle of FEA is that it breaks a very complex problem into many simpler problems that are much easier to solve. It does this by breaking down complex shapes and geometries into simple shapes called elements. In this way, complex systems such as atheroma can be modeled appropriately and structural stresses can 
be quantified. Because FEA integrates information regarding plaque morphology, material properties of the plaque components and patient-specific blood pressure (used for loading) during the computational simulations, it provides a much more comprehensive assessment of plaque vulnerability compared to plaque morphology alone. The present study, however, has some limitations.

Relative material properties of various plaque components were used, which are probably only an approximate representation of the actual material properties but they are the best available choice for the biomechanical simulations. This limitation is difficult to overcome at present. Second, conventional black-blood imaging sequences were used to identify hemorrhage. Very recently Ota et al reported that the 3-D magnetization-prepared rapid-acquisition gradient-echo (RAGE) sequence demonstrates higher diagnostic capability for the detection and quantification of $\mathrm{PH}$ compared to 2-D fast spin-echo and 3-D TOF sequences. ${ }^{39}$ Because fast spin echo sequences were used in the present study, it is possible that we could have underestimated the size of $\mathrm{PH}$ in the present study compared to what could have been achieved by using the RAGE sequence ${ }^{39}$ Perhaps for future studies this sequence may be used for better PH characterization. MRI comparison/ co-registration was not done, because this has been validated by us previously. ${ }^{40}$ Finally, transthoracic echocardiography was used to exclude cardiogenic source of thrombus. Ideally it is better to use trans-esophageal echocardiography and also perform strict exclusion of another thrombogenic source by performing imaging from aortic root to the aortic arch and proximal carotid arteries.

\section{Conclusions}

The present study suggests that MRI-based FEA of plaques has the ability to assess and differentiate the stress profiles of hemorrhagic and non-hemorrhagic plaques. Hemorrhagic plaques were observed to have high stresses. From previous studies, we know that hemorrhagic plaques are associated with thromboembolic events. This technique therefore appears to have the potential to identify high-risk plaques and may be useful in refining risk stratification criteria in future.

\section{Acknowledgments}

Dr Umar Sadat is supported by a Medical Research Council UK and Royal College of Surgeons of England Joint Clinical Research Training Fellowship. This research has also been supported by Biomedical Research Centre National Institute of Health Research (BRC NIHR) grant.

\section{References}

1. Falk E. Unstable angina with fatal outcome: Dynamic coronary thrombosis leading to infarction and/or sudden death: Autopsy evidence of recurrent mural thrombosis with peripheral embolization culminating in total vascular occlusion. Circulation 1985; 71: 699708 .

2. Altaf N, Daniels L, Morgan PS, Auer D, MacSweeney ST, Moody $\mathrm{AR}$, et al. Detection of intraplaque hemorrhage by magnetic resonance imaging in symptomatic patients with mild to moderate carotid stenosis predicts recurrent neurological events. J Vasc Surg 2008; 47: $337-342$.

3. Altaf N, MacSweeney ST, Gladman J, Auer DP. Carotid intraplaque hemorrhage predicts recurrent symptoms in patients with high-grade carotid stenosis. Stroke 2007; 38: $1633-1635$.

4. Altaf N, Daniels L, Morgan PS, Lowe J, Gladman J, MacSweeney ST, et al. Cerebral white matter hyperintense lesions are associated with unstable carotid plaques. Eur J Vasc Endovasc Surg 2006; 31: $8-13$.

5. Imparato AM, Riles TS, Gorstein F. The carotid bifurcation plaque: Pathologic findings associated with cerebral ischemia. Stroke 1979;
10: $238-245$.

6. Takaya N, Yuan C, Chu B, Saam T, Polissar NL, Jarvik GP, et al. Presence of intraplaque hemorrhage stimulates progression of carotid atherosclerotic plaques: A high-resolution magnetic resonance imaging study. Circulation 2005; 111: 2768-2775.

7. Gao P, Chen ZQ, Bao YH, Jiao LQ, Ling F. Correlation between carotid intraplaque hemorrhage and clinical symptoms: Systematic review of observational studies. Stroke 2007; 38: 2382-2390.

8. Lusby RJ, Ferrell LD, Ehrenfeld WK, Stoney RJ, Wylie EJ. Carotid plaque hemorrhage: Its role in production of cerebral ischemia. Arch Surg 1982; 117: 1479-1488.

9. Lusby RJ, Woodcock JP, Machleder HI, Ferrell LD, Jeans WD, Skidmore R, et al. Transient ischaemic attacks: The static and dynamic morphology of the carotid artery bifurcation. Br J Surg 1982; 69(Suppl): S41-S44.

10. Li ZY, Howarth SP, Tang T, Graves MJ, U-King-Im J, Trivedi RA, et al. Structural analysis and magnetic resonance imaging predict plaque vulnerability: A study comparing symptomatic and asymptomatic individuals. J Vasc Surg 2007; 45: 768-775.

11. Sadat U, Li ZY, Young VE, Graves MJ, Boyle JR, Warburton EA, et al. Finite element analysis of vulnerable atherosclerotic plaques: A comparison of mechanical stresses within carotid plaques of acute and recently symptomatic patients with carotid artery disease. J Neurol Neurosurg Psychiatry 2010; 81: 286-289.

12. Sadat U, Teng Z, Young VE, Walsh SR, Li ZY, Graves MJ, et al. Association between biomechanical structural stresses of atherosclerotic carotid plaques and subsequent ischaemic cerebrovascular events: A longitudinal in vivo magnetic resonance imaging-based finite element study. Eur J Vasc Endovasc Surg 2010; 40: 485-491.

13. Zhu C, Teng Z, Sadat U, Young VE, Graves MJ, Li ZY, et al. Normalized wall index specific and MRI-based stress analysis of atherosclerotic carotid plaques: A study comparing acutely symptomatic and asymptomatic patients. Circ J 2010; 74: 2360-2364.

14. Eliasziw M, Kennedy J, Hill MD, Buchan AM, Barnett HJ. Early risk of stroke after a transient ischemic attack in patients with internal carotid artery disease. CMAJ 2004; 170: 1105-1109.

15. Mitsumori LM, Hatsukami TS, Ferguson MS, Kerwin WS, Cai J, Yuan C. In vivo accuracy of multisequence MR imaging for identifying unstable fibrous caps in advanced human carotid plaques. J Magn Reson Imaging 2003; 17: 410-420.

16. Saam T, Cai J, Ma L, Cai YQ, Ferguson MS, Polissar NL, et al. Comparison of symptomatic and asymptomatic atherosclerotic carotid plaque features with in vivo MR imaging. Radiology 2006; 240: 464-472.

17. Howarth SP, Tang TY, Trivedi R, Weerakkody R, U-King-Im J, Gaunt ME, et al. Utility of USPIO-enhanced MR imaging to identify inflammation and the fibrous cap: A comparison of symptomatic and asymptomatic individuals. Eur J Radiol 2009; 70: 555-560.

18. Yuan C, Mitsumori LM, Ferguson MS, Polissar NL, Echelard D, Ortiz $\mathrm{G}$, et al. In vivo accuracy of multispectral magnetic resonance imaging for identifying lipid-rich necrotic cores and intraplaque hemorrhage in advanced human carotid plaques. Circulation 2001; 104: $2051-2056$.

19. Sadat U, Weerakkody RA, Bowden DJ, Young VE, Graves MJ, Li $\mathrm{ZY}$, et al. Utility of high resolution MR imaging to assess carotid plaque morphology: A comparison of acute symptomatic, recently symptomatic and asymptomatic patients with carotid artery disease. Atherosclerosis 2009; 207: 434-439.

20. Oikawa M, Ota H, Takaya N, Miller Z, Hatsukami TS, Yuan C. Carotid magnetic resonance imaging: A window to study atherosclerosis and identify high-risk plaques. Circ J 2009; 73: 1765-1773.

21. Yang C, Tang D, Yuan C, Hatsukami TS, Zheng J, Woodard PK. In vivo/ex vivo MRI-based 3D non-Newtonian FSI models for human atherosclerotic plaques compared with fluid/wall-only models. Comput Model Eng Sci 2007; 19: 233-246.

22. Tang D, Teng Z, Canton G, Yang C, Ferguson M, Huang X, et al. Sites of rupture in human atherosclerotic carotid plaques are associated with high structural stresses: An in vivo MRI-based 3D fluidstructure interaction study. Stroke 2009; 40: 3258-3263.

23. Bathe KJ. Theory and modeling guide. Watertown, MA: ADINA R\&D, 2002.

24 Teng Z, Sadat U, Li Z, Huang X, Zhu C, Young VE, et al. Arterial luminal curvature and fibrous-cap thickness affect critical stress conditions within atherosclerotic plaque: An in vivo MRI-based 2D finite-element study. Ann Biomed Eng 2010; 38: 3096-3101.

25. Gao H, Long Q, Graves M, Gillard JH, Li ZY. Study of reproducibility of human arterial plaque reconstruction and its effects on stress analysis based on multispectral in vivo magnetic resonance imaging. J Magn Reson Imaging 2009; 30: 85-93.

26. Tang D, Teng Z, Canton G, Hatsukami TS, Dong L, Huang X, et al. 
Local critical stress correlates better than global maximum stress with plaque morphological features linked to atherosclerotic plaque vulnerability: An in vivo multi-patient study. Biomed Eng Online 2009; 8: 15 .

27. U-King-Im JM, Tang TY, Patterson A, Graves MJ, Howarth S, Li $\mathrm{ZY}$, et al. Characterisation of carotid atheroma in symptomatic and asymptomatic patients using high-resolution MRI. J Neurol Neurosurg Psychiatry 2008; 79: 905-912.

28. Javid H, Ostermiller WE, Hengesh JW, Dye WS, Hunter JA, Najafi $\mathrm{H}$, et al. Carotid endarterectomy for asymptomatic patients. Arch Surg 1971; 102: 389-391.

29. Levy AP, Moreno PR. Intraplaque hemorrhage. Curr Mol Med 2006; 6: $479-488$.

30. Lusby RJ, Machleder HI, Jeans W, Skidmore R, Woodcock JP, Clifford PC, et al. Vessel wall and blood flow dynamics in arterial disease. Philos Trans R Soc Lond B Biol Sci 1981; 294: 231-239.

31. Redgrave JN, Lovett JK, Gallagher PJ, Rothwell PM. Histological assessment of 526 symptomatic carotid plaques in relation to the nature and timing of ischemic symptoms: The Oxford plaque study. Circulation 2006; 113: 2320-2328.

32. Li ZY, Howarth S, Trivedi RA, U-King-Im JM, Graves MJ, Brown $\mathrm{A}$, et al. Stress analysis of carotid plaque rupture based on in vivo high resolution MRI. J Biomech 2006; 39: 2611-2622.

33. Li ZY, Howarth S, U-King-Im J, Gillard J. Atheroma: Is calcium important or not? A modelling study of stress within the atheromatous fibrous cap in relation to position and size of calcium deposits.
Conf Proc IEEE Eng Med Biol Soc 2005; 3: 2236-2239.

34. Li ZY, Howarth S, Tang T, Graves M, U-King-Im J, Gillard JH. Does calcium deposition play a role in the stability of atheroma? Location may be the key. Cerebrovasc Dis 2007; 24: 452-459.

35. Li ZY, Howarth SP, Tang T, Gillard JH. How critical is fibrous cap thickness to carotid plaque stability? A flow-plaque interaction model. Stroke 2006; 37: 1195-1199.

36. Tang TY, Howarth SP, Li ZY, Miller SR, Graves MJ, U-King-Im $\mathrm{JM}$, et al. Correlation of carotid atheromatous plaque inflammation with biomechanical stress: Utility of USPIO enhanced MR imaging and finite element analysis. Atherosclerosis 2008; 196: 879-887.

37. Trivedi RA, Li ZY, U-King-Im J, Graves MJ, Kirkpatrick PJ, Gillard JH. Identifying vulnerable carotid plaques in vivo using high resolution magnetic resonance imaging-based finite element analysis. J Neurosurg 2007; 107: 536-542.

38. Li ZY, Tang T, U-King-Im J, Graves M, Sutcliffe M, Gillard JH. Assessment of carotid plaque vulnerability using structural and geometrical determinants. Circ J 2008; 72: 1092-1099.

39. Ota H, Yarnykh VL, Ferguson MS, Underhill HR, Demarco JK, Zhu DC, et al. Carotid intraplaque hemorrhage imaging at 3.0-T MR imaging: Comparison of the diagnostic performance of three T1-weighted sequences. Radiology 2010; 254: 551-563.

40. Trivedi RA, U-King-Im JM, Graves MJ, Horsley J, Goddard M, Kirkpatrick PJ, et al. MRI-derived measurements of fibrous-cap and lipid-core thickness: The potential for identifying vulnerable carotid plaques in vivo. Neuroradiology 2004; 46: 738-743. 